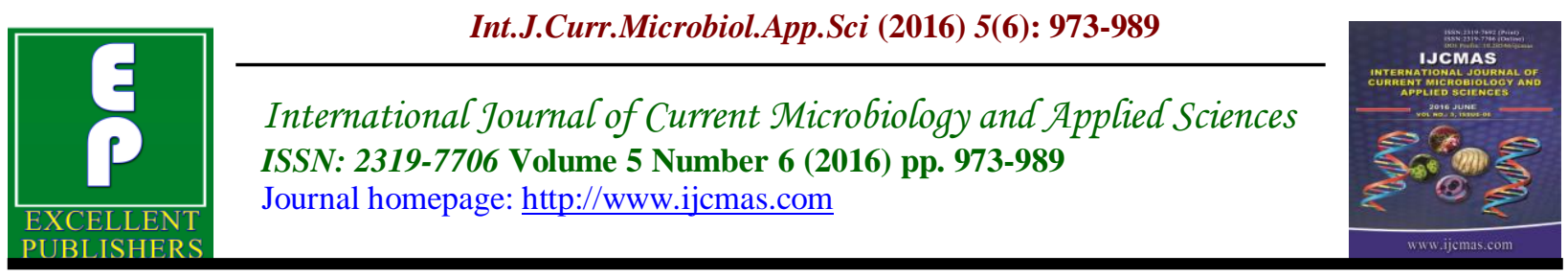

Original Research Article

http://dx.doi.org/10.20546/ijcmas.2016.506.104

\title{
Phytodiversity of Ganpur forest, Birbhum District, West Bengal, India with reference to their Medicinal properties
}

\author{
Nisith Ranjan Sarkar, Subrata Mondal and Sudhendu Mandal* \\ UGC-DRS Department of Botany, Visva-Bharati, Santiniketan- 731235, India \\ *Corresponding author
}

\begin{tabular}{ll}
\hline & A B S T R A C T \\
\cline { 2 - 2 } $\begin{array}{l}\text { Keywords } \\
\begin{array}{l}\text { Phytodiversity, } \\
\text { conservation, } \\
\text { biodiversity, }\end{array}\end{array}$ & $\begin{array}{l}\text { The present study deals with the observation on phytodiversity including its } \\
\text { uses, distribution and occurrence to achieve the current strategic plan for } \\
\text { biodiversity conservation. The plants are enumerated according to the } \\
\text { alphabetical order of the plant names along with local names and their uses. }\end{array}$ \\
$\begin{array}{ll}\text { Gonpur forest } \\
\text { Article Info }\end{array}$ & $\begin{array}{l}\text { During this investigation } 229 \text { plant species were collected from the forest } \\
\text { under } 81 \text { families and } 184 \text { genera, among them } 193 \text { species belong to } \\
\text { dicots with } 70 \text { families and } 154 \text { genera, and the } 11 \text { families } 30 \text { genera and }\end{array}$ \\
$\begin{array}{ll}\text { Accepted: } \\
\text { 28 May 2016 } \\
\begin{array}{l}\text { Available Online: } \\
\text { 10 June 2016 }\end{array}\end{array}$ & $\begin{array}{l}\text { like Jharkhand identified Gonpur forest as one of the major threats to } \\
\text { vegetation and flora of the forest. }\end{array}$ \\
\hline
\end{tabular}

\section{Introduction}

The Birbhum district is one of the lateritic belt district of West Bengal. It is situated between $23^{\circ} 32^{\prime} 30^{\prime \prime}$ to $24^{\circ} 35^{\prime \prime} 00^{\prime \prime}$ North latitude and $87^{\circ} 5^{\prime} 25^{\prime \prime}$ to $88^{\circ} 2^{\prime}$ East longitude, total area of the district is about 4550.94 sq.km $(5.10 \%$ of the state area). The climate of Birbhum district may be said as "dry sub-humid mega thermal" (Thornthwaite, 1948). Maximum temperature rise up to $44^{\circ} \mathrm{c}-46^{\circ} \mathrm{c}$ during April-May and goes down to $6^{\circ}-7^{\circ} \mathrm{c}$ during December-January. Rainfall is moderate, maximum of $350 \mathrm{~mm}$ during July. The present study area Ganpur forest range situated between 2404'37.0"$24^{\circ} 04^{\prime} 37.5^{\prime \prime l a t i t u d e}$ and $087^{\circ} 40^{\prime} 59.1^{\prime \prime}$ to $087^{\circ} 41^{\prime} 004^{\prime \prime}$ longitude.
It is one of the most species rich forests in this district that is still unexplored. Few scattered works on the flora of Birbhum district have been published by Basak, 1968 \& 75; Guha, 1968; Basak and Guha Bakshi, 1977; Chowdhury and Mandal, 1999. Few works on ethno-medicinal plants and phytosociological study of plant species has been done from this district (Rahman and Mandal,1999; Bhattacharyaa et al.,2003; Rahaman et al, 2008 \& 2015). The medicinal uses of plant species reported by the tribes were compared and thoroughly screened with important works such as Kirtikar and Basu (1933), Chopra et al. $(1969,1956)$, The wealth of India(1948-1976) and Jain(1991). 
It has been found that different tribal communities like Santhal, Konra, etc. live in the village named Choubata, Kanta Pahari, Oal Pahari, Kolai Pahari, Ghanga etc. attached to the forest. Various valuable medicinal plants are found here and tribal people of the surrounding area are very much familiar with those plants as they use those plants to produce their herbal medicines for treatment of various diseases. Not only that, healers from Jharkhand and the local healers come to the forest during Monsoon for harvesting huge amount of medicinally important plant specimen and sell them in the market. The plant bank reports to know the health of the forest and the uses of the plant from this forest for medicinal purposes and other purposes which are important for the benefit of humankind. This work will also document the different plant species of the from the conservation point of view.

\section{Materials and Methods}

Extensive surveys for a period of three years (April 2012 to March 2015) in the forest have been made at regular intervals. The plant specimens were collected and identified by using standard monographs and flora (Prain 1903, Hooker 1897) and consultation from the Visva-Bharati Herbarium (VBH), Santiniketan as well as from Central National Herbarium (CAL), Sibpur, Howrah. The collected plant specimens are properly processed and herbarium sheets have been made and preserved as herbarium specimen following the standard method (Jain and Rao, 1977). Various information regarding the uses of the plant parts have been collected on the basis of frequent interviews with the tribal guru to know the practicing indigenous knowledge of medicine and documented in the field note book and confirmed repeated interviews at other places (Table-2).
Vegetation pattern survey was carried out during April 2012 to March 2015 by laying $10 \mathrm{~m} \times 10 \mathrm{~m}$ quadrates at 50 spots and was sampled through quadrates placed randomly; number of each species has been noted.

\section{Results and Discusion}

229 plant species of 81 families represented by 184 genera have been enumerated and documented in alphabetical order of the plant names (Table-1). The medicinally important plants as per information given by the Tribal Gurus are also enumerated (Table-2). Collected plant specimens are statistically classified and found 193 Dicotyledonous species under 70 families and 36 Monocotyledonous species under 11 families (Table-5). Collected species are properly processed for herbarium and labelled them properly with collection Number, Field No. etc. arranged according to the classification system of Bentham and Hooker (1887-97) and deposited in the Visva Bharati Herbarium(VBH) of Botany Department, Visva-Bharati, Santiniketan, West-Bengal, India.

Ganpur forest is dominated by Shorea robusta Gaertn.f. (Dipterocarpaceae), Madhuca indica J.F. Gmel.(Sapotaceae), Terminalia bellirica Roxb.(Combretaceae), Acacia auriculiformis (Mimosaceae) etc. Eleven most dominant families in the Gonpur forest are Fabaceae $(18.75 \%)$ with 15 taxa in the first under 11 genus, followed by Acanthaceae $(17.5 \%$ ) with 14 taxa under 9 genus, Poaceae (20.0\%)with 16 taxa under13 genus, Euphorbiaceae (14.8\%) with 12 taxa under 8 genus, Asteraceae $(13.6 \%)$ with 11 taxa under 11 genus, Rubiaceae (13.6\%) with 11 taxa under 11 genus, Scrophulariaceae $(11.1 \%)$ with 9 taxa under 6 genus, Caesalpiniaceae $(7.4 \%)$ with 6 taxa under 3 genus, Lamiaceae $(7.4 \%)$ with 6 taxa under 5 genus, 
Apocynaceae (6.1\%) with 5 taxa under 5 genus, Amaranthaceae (6.1\%) with 5 taxa under 4 genus, Verbenaceae $(6.1 \%)$ with 5 taxa under 5 genus and Convovulaceae (6.1\% ) also recorded 5 taxa under 4 genus (Table-3);(Fig-1). Analysis of the life form composition shows that herbaceous taxa of 116 occupied $50.66 \%$ of the flora, 55 taxa of trees $(24.02 \%), 42$ taxa of shrubs ( 18.34 $\%)$ and 16 taxa of climbers (6.99\%) (Table- 4) (Fig-2).

Table.1 Plant species enumeration of Ganpur forest, Birbhum District

\begin{tabular}{|c|c|c|c|c|c|c|}
\hline Sl. No. & Scientific name of the plants & Habits & Family & Local name & Availability & \begin{tabular}{|l} 
Flowering\& \\
Fruiting time
\end{tabular} \\
\hline 1. & Abrus precatorius $\mathrm{L}$. & $\mathrm{S}$ & Fabaceae & Kunch & Less common & Aug.-Sep. \\
\hline 2. & Abutilon indicum (L.) Sweet & $\mathrm{S}$ & Malvaceae & Bon kapas & Common & May-Aug. \\
\hline 3. & Acacia auriculiformis A.Cunn.ex Benth. & $\mathrm{T}$ & Mimosaceae & $\begin{array}{l}\text { Sonajhuri/ } \\
\text { Akashmoni }\end{array}$ & Common & Sep.-Nov. \\
\hline 4. & Acacia nilotica Dellile. & $\mathrm{T}$ & Mimosaceae & Babla & Common & Aug.-Mar. \\
\hline 5. & Achyranthes aspera $\mathrm{L}$. & $\mathrm{H}$ & Amaranthaceae & Apang & Common & Aug-Feb. \\
\hline 6. & Adina cordifolia (Roxb.) Brandis. & $\mathrm{T}$ & Rubiaceae & Chakalta & Not common & May.-Aug. \\
\hline 7. & Aegle marmelos (L.) Correa. & $\mathrm{T}$ & Rutaceae & Bel & rare & Apr.-Jul. \\
\hline 8. & Aerva lanata (L.) Juss.ex Schult. & $\mathrm{H}$ & Amaranthaceae & $\begin{array}{l}\text { Jaya phul/ } \\
\text { daya phul }\end{array}$ & Common & Jul.-Apr. \\
\hline 9. & Agave americana $\mathrm{L}$. & $\mathrm{S}$ & Agavaceae & Konga & Less common & Dec.-Jan. \\
\hline 10. & Ageratum conyzoides Sieber ex Steud. & $\mathrm{H}$ & Asteraceae & Not known & Common & Jun.-Jan. \\
\hline 11. & Alangium salviifolium (L.f.) Wangerin & $\mathrm{T}$ & Alangiaceae & Ankor & Common & Mar.-May \\
\hline 12. & Albizia lebbeck (L.) Benth. & $\mathrm{T}$ & Mimosaceae & Sirish & Common & Apr.-Mar. \\
\hline 13. & Alstonia scholaris (L.) R.Br. & $\mathrm{T}$ & Apocynaceae & Chhatim & Less common & Dec.-Apr. \\
\hline 14. & Alysicarpus monilifer (L.) DC. & $\mathrm{H}$ & Fabaceae & Not known & Less common & Aug.-Apr. \\
\hline 15. & Amaranthus spinosus.L. & $\mathrm{H}$ & Amaranthaceae & Kantanotey & common & Whole year \\
\hline 16. & Amaranthus viridis Pollich ex Moq. & $\mathrm{H}$ & Amaranthaceae & Notey & Common & Whole year \\
\hline 17. & Anacardium occidentale $\mathrm{L}$. & $\mathrm{T}$ & Anacardiaceae & Kaju badam & Less common & Feb.-Apr. \\
\hline 18. & Andrographis paniculata Nees. & $\mathrm{H}$ & Acanthaceae & kalmegh & Common & Sep.-Dec.. \\
\hline 19. & Anisomeles ovata R.Br. & $\mathrm{S}$ & Lamiaceae & Apang & Less common & Sep.-Dec. \\
\hline 20. & Annona reticulata Vell. & $\mathrm{T}$ & Annonaceae & Nona-Ata & Less common & May-Jul. \\
\hline 21. & Antigonon leptopus Hook.\&Arn. & $\mathrm{CL}$ & Polygonaceae & Anantalata & Common & Jul.-Nov. \\
\hline 22. & Argyreia nervosa (Burm.f.)Bojer & $\mathrm{CL}$ & Convolvulaceae & Gogul & Not common & Sep.-Jan. \\
\hline 23. & Aristida setaceae Retz. & $\mathrm{H}$ & Poaceae & Not known & Common & Sep.-Jan. \\
\hline 24. & Aristolochia indica L. & $\mathrm{H}$ & Aristolochiaceae & Iswarmul & Less common & Jun.-Oct. \\
\hline 25. & Asparagus racemosus Willd. & $\mathrm{CL}$ & Liliaceae & Satamuli & Common & Sep.-Oct. \\
\hline 26. & Azadirachta indica A.Juss. & $\mathrm{T}$ & Meliaceae & Neem & Common & Mar.-May. \\
\hline 27. & Barleria cristata Lam. & $\mathrm{S}$ & Acanthaceae & Janti & Not common & Sep.-Jan. \\
\hline 28. & Barleria prionitis L. & $\mathrm{S}$ & Acanthaceae & Kantanotey & Not common & Sep.-Apr. \\
\hline 29. & Bixa orellana $\mathrm{L}$. & $\mathrm{T}$ & Bixaceae & Latkan & Less common & Jul.-Oct. \\
\hline 30. & Blumea oxyodonta DC. & $\mathrm{H}$ & Asteraceae & Not known & Common & Dec.-Mar. \\
\hline 31. & Boerhavia diffusa Engehm.\& A.Gray & $\mathrm{H}$ & Nyctaginaceae & Punornova & Common & Oct.-Apr. \\
\hline 32. & Borassus flabellifer L. & $\mathrm{T}$ & Arecaceae & Tal & Common & Feb-Apr.. \\
\hline
\end{tabular}




\begin{tabular}{|c|c|c|c|c|c|c|}
\hline 33. & Borreria articularis F.N.Williams. & $\mathrm{T}$ & Rubiacea & Not known & Common & Jul.-Jan. \\
\hline 34. & Bridelia retusa Spreng. & $\mathrm{T}$ & Euphorbiaceae & Bhalas/ Geio & Common & Aug.-Nov. \\
\hline 35. & Bryophyllum calycinum Salisb. & $\mathrm{T}$ & Crassulaceae & patharkuchi & Not common & Mar.-Apr. \\
\hline 36. & Buchanania lanzan Spreng. & $\mathrm{T}$ & Anacardiaceae & Piyal & Less common & Jan.-Feb. \\
\hline 37. & Butea monosperma (Lam.) Taub. & $\mathrm{T}$ & Fabaceae & Lal palash & Common & Feb-Jun. \\
\hline 38. & Butea superba Roxb.ex.Wild. & $\mathrm{H}$ & Fabaceae & Latano-palash & Rare & Feb.-July \\
\hline 39. & Canscora diffusa R.Br. & $\mathrm{H}$ & Gentianaceae & Not known & Common & Oct.-Jan. \\
\hline 40. & Cardiospermum halicacabum $\mathrm{L}$. & $\mathrm{H}$ & Sapindaceae & Sibghul & Common & Whole year \\
\hline 41. & Carissa spinarum Lodd.ex.A.DC. & $\mathrm{S}$ & Apocynaceae & $\begin{array}{l}\text { Buno } \\
\text { karamcha }\end{array}$ & Common & Mar.-May. \\
\hline 42. & Cassia fistula L. & $\mathrm{T}$ & Caesalpiniaceae & $\begin{array}{l}\text { Bandarlathi/A } \\
\text { maltas }\end{array}$ & Common & Apr.-May \\
\hline 43. & Centella asiatica Urb. & $\mathrm{H}$ & Apiaceae & Thankuni & Common & Apr.-Jul. \\
\hline 44. & Chenopodium album Bosc.ex Moq. & $\mathrm{H}$ & Chenopdiaceae & Bethosak & Common & Nov.-Mar. \\
\hline 45. & Chrysopogon aciculatus Trin. & $\mathrm{H}$ & Poaceae & Chorkanta & Common & Aug.-Oct. \\
\hline 46. & Chrysopogon lancearius Haines. & $\mathrm{H}$ & Poaceae & Not known & Common & Sep.-Oct. \\
\hline 47. & Cissus quadrangularis L. & $\mathrm{CL}$ & Vitaceae & Harjora & Common & Feb.-Oct. \\
\hline 48. & Cleome viscosa L. & $\mathrm{H}$ & Capparidaceae & Harhura & Common & Jul.-Sep. \\
\hline 49. & Clerodendrum petasites S.Moore & $\mathrm{S}$ & Verbenaceae & Not known & common & Jan.-Mar. \\
\hline 50. & Coccinia grandis (L.) Voigt & $\mathrm{CL}$ & Cucurbitaceae & Telakucha & Common & Aug.-Oct. \\
\hline 51. & Coix lacryma-jobi $\mathrm{L}$. & $\mathrm{H}$ & Poaceae & Garagra & Rare & Sept.-Oct. \\
\hline 52. & Colocasia esculenta $(\mathrm{L}$.)Schott & $\mathrm{H}$ & Araceae & Kachu & Common & July.-Nov. \\
\hline 53. & Commelina benghalensis L. & $\mathrm{H}$ & Commelinaceae & Kanshira & Very common & Jul.-Nov. \\
\hline 54. & Corchorus aestuans Herb.Madr.ex Wall. & $\mathrm{H}$ & Tiliaceae & Tita-pat & Less common & Jul.-Aug. \\
\hline 55. & Corchorus fascicularis Lam. & $\mathrm{H}$ & Tiliaceae & Jangli-pat & Less common & Jul.-Sep. \\
\hline 56. & Costus speciosus (J.Koenig.) Sm. & $\mathrm{H}$ & Zingiberaceae & Not known & Not common & Jul.-Sep. \\
\hline 57. & Crotalaria prostrata Rottler. & $\mathrm{H}$ & Fabaceae & Jhunjhuni & Common & Sep.-Dec. \\
\hline 58. & Crotalaria retusa $\mathrm{L}$. & $\mathrm{H}$ & Fabaceae & Atasi & Common & Whole year \\
\hline 59. & Crotalaria verrucosa $\mathrm{L}$. & $\mathrm{S}$ & Fabaceae & Bon-son & Not common & Oct.-Jan. \\
\hline 60. & Curculigo orchioides Gaertn. & $\mathrm{H}$ & Hypoxideae & Kali Musali & Not common & Aug.-Nov. \\
\hline 61. & Curcuma aromatica Salisb. & $\mathrm{H}$ & Zingiberaceae & Bon Halud & Rare & May.-Jun. \\
\hline 62. & Cuscuta reflexa Roxb. & CL & Cuscutaceae & Swarnalata & Less common & Oct.-Feb. \\
\hline 63. & Cyanotis tuberosa (Roxb.) Schult. f. & $\mathrm{H}$ & Commelinaceae & Not known & Less common & Jul.-Oct. \\
\hline 64. & Cymbopogon martini Staef. & $\mathrm{H}$ & Poaceae & Not known & Rare & Oct-Dec. \\
\hline 65. & Cynodon dactylon (L.) Pers & $\mathrm{H}$ & Poaceae & Durba & Common & Sep.-Feb. \\
\hline 66. & Cyperus difformis(L.) & $\mathrm{H}$ & Cyperaceae & Not known & Common & Aug.-Dec. \\
\hline 67. & Cyperus monocephalus Baker. & $\mathrm{H}$ & Cyperaceae & Not known & Common & Jun.-Jan. \\
\hline 68. & Cyperus rotundus Kunth. & $\mathrm{H}$ & Cyperaceae & Mutha & Common & Jun.-Jan. \\
\hline 69. & Dactyloctenium aegyptium (L.)K.Richt. & $\mathrm{H}$ & Poaceae & Not known & Common & July.-Dec. \\
\hline 70. & Dalbergia sissoo. Roxb. & $\mathrm{T}$ & Fabaceae & Sissu & Common & Mar.Aug. \\
\hline 71. & Dendrophthoe falcata Blume & $\mathrm{H}$ & Loranthaceae & Banda & Common & Nov.-Mar. \\
\hline 72. & Dentella repens J.R.Forst \& G.Forst. & $\mathrm{H}$ & Rubiaceae & Not known & Common & Sep.-Feb. \\
\hline 73. & Desmodium gangeticum (L.) DC. & $\mathrm{H}$ & Fabaceae & Salpani & Common & May.-Jan. \\
\hline
\end{tabular}




\begin{tabular}{|c|c|c|c|c|c|c|}
\hline 74. & Desmodium triflorum (L.) DC. & $\mathrm{H}$ & Fabaceae & Kudali & & Sep.-Apr. \\
\hline 75. & Digitaria ciliaris (Retz.) Koeler. & $\mathrm{H}$ & Poaceae & Makur-jali & Common & Aug.-Oct. \\
\hline 76. & Dillenia pentagyna Roxb. & $\mathrm{T}$ & Dilleniaceae & Bon chalta & Rare & Mar.-Jun. \\
\hline 77. & Dioscorea alata $\mathrm{L}$. & $\mathrm{CL}$ & Diascoreaceae & Khamalu & Not common & July - Oct. \\
\hline 78. & Dioscorea bulbifera $\mathrm{L}$. & $\mathrm{CL}$ & Diascoreaceae & Kukuralu & Less common & Sep.-Oct \\
\hline 79. & Diospyros melanoxylon Hiem. & $\mathrm{T}$ & Ebenaceae & Kend & Rare & Apr.-Jun \\
\hline 80. & Dipterocanthus proatratus (Poir.) & $\mathrm{H}$ & Acanthaceae & Not known & Not common & Jun.-Oct. \\
\hline 81. & Drosera burmanni DC. & $\mathrm{H}$ & Droseraceae & Surjasisir & Rare & Oct.-Jan. \\
\hline 82. & Echinochloa colona (L.) Link & $\mathrm{H}$ & Poaceae & Not known & Very common & Jul.-Oct. \\
\hline 83. & Eclipta prostrata (L.) & $\mathrm{H}$ & Asteraceae & $\begin{array}{l}\text { Bhringaraj/ } \\
\text { keshute }\end{array}$ & Common & Whole year \\
\hline 84. & Elephantopus scaber L. & $\mathrm{H}$ & Asteraceae & Hastipod & Rare & Sep.-Dec. \\
\hline 85. & Eleusine indica (L.)Gaertn. & $\mathrm{H}$ & Poaceae & Sursuri ghas & Common & Aug.-Nov. \\
\hline 86. & Emilia sonchifolia $(\mathrm{L}$.$) DC.$ & $\mathrm{H}$ & Asteraceae & Sadimodi & Less common & Nov.-Feb. \\
\hline 87. & Eragrostis coarctata Stapf & $\mathrm{H}$ & Poaceae & Not known & Common & Aug.-Feb. \\
\hline 88. & Eragrostis tenella Benth. & $\mathrm{H}$ & Poaceae & Sursuri ghas & Common & Jul.-Jan. \\
\hline 89. & Eriocaulon quinquangulare L. & $\mathrm{H}$ & Eriocaulaceae & Not known & Common & Sept.-Jan. \\
\hline 90. & Eucalyptus citriodora Hook. & $\mathrm{T}$ & Myrtaceae & Eucalyptus & Common & Sep.-May. \\
\hline 91. & Eupatorium odoratum $\mathrm{L}$. & $\mathrm{H}$ & Asteraceae & Not known & Common & Nov.Jan. \\
\hline 92. & Euphorbia antiquorum E.Mey. & $\mathrm{S}$ & Euphorbiaceae & $\begin{array}{l}\begin{array}{l}\text { Teshare } \\
\text { monsa }\end{array} \\
\end{array}$ & Rare & Dec.-Jan. \\
\hline 93. & Euphorbia hirta L. & $\mathrm{H}$ & Euphorbiaceae & Barokarni & Common & Whole year \\
\hline 94. & Evolvulus alsinoides L. & $\mathrm{H}$ & Convolvulaceae & Not known & Common & July.-Nov. \\
\hline 95. & Evolvulus nummularius (L.) L. & $\mathrm{H}$ & Convolvulaceae & Not known & Common & Whole year \\
\hline 96. & Ficus benghalensis L. & $\mathrm{T}$ & Moraceae & Bot & Common & Apr.Jul. \\
\hline 97. & Ficus hispida L.f. & $\mathrm{T}$ & Moraceae & Dumur & Common & Feb.-Mar. \\
\hline 98. & Ficus racemosa Wall. & $\mathrm{T}$ & Moraceae & Jagnya dumur & Not common & Apr.-Jul. \\
\hline 99. & Ficus religiosa Decne.ex Miq. & $\mathrm{T}$ & Moraceae & Aswathha & Common & $\begin{array}{l}\text { Apr.-May \& } \\
\text { Oct.-Nov. }\end{array}$ \\
\hline 100. & Fimbristylis teragona $\mathrm{R} . \mathrm{Br}$. & $\mathrm{H}$ & Cyperaceae & Not known & Less common & Sep.-Jan. \\
\hline 101. & Flacourtia jangomas (Lour.) Raeusch. & $\mathrm{S}$ & Flacourtiaceae & Bainchi & Rare & Mar-.May \\
\hline 102. & Flacourtia indica (Burm.f.)Merr. & $\mathrm{T}$ & Flacourtiaceae & Bainchi & Common & Jan.-Mar. \\
\hline 103. & Glinus oppositifolius (L.) Aug.DC. & $\mathrm{H}$ & Aizoaceae & Gima & Common & Jul.Oct. \\
\hline 104. & Globba bulbifera Roxb. & $\mathrm{H}$ & Zingiberaceae & Not known & Not common & Aug.-Sep. \\
\hline 105. & Glochidion lanceolarium Voigt. & $\mathrm{T}$ & Euphorbiaceae & Not known & Not common & Feb-Mar. \\
\hline 106. & Gloriosa superba L. & $\mathrm{CL}$ & Liliaceae & Ulat-chandal & Less common & Jun.-Sep. \\
\hline 107. & Gnaphalium luteo-album L. & $\mathrm{H}$ & Asteraceae & Not known & Common & Mar.-Jun. \\
\hline 108. & Gomphrena celosioides Mart, & $\mathrm{H}$ & Amaranthaceae & Not known & Common & Mar.-Sep. \\
\hline 109. & Grewia hirsute Roxb. & $\mathrm{T}$ & Tiliaceae & Kukurbicha & Less common & Jul.-Sep. \\
\hline 110. & Gymnema sylvestre (Retz.) R.Br.ex Sm & $\mathrm{CL}$ & Asclepiadaceae & Gurmari & Rare & Aug.-Oct. \\
\hline 111. & Hedyotis pinifolia Wall. & $\mathrm{H}$ & Rubiaceae & Not known & Not common & Jul.-Feb. \\
\hline 112. & Heliotropium indicum $\mathrm{L}$. & $\mathrm{H}$ & Boraginaceae & Hatisur & Common & May.-Dec. \\
\hline 113. & Hemidesmus indicus (L.) R.Br. & $\mathrm{H}$ & Asclepiadaceae & Anantamul & Common & Aug.-Dec. \\
\hline 114. & Hemigraphis hirta T.Anderson & $\mathrm{H}$ & Acanthaceae & Not known & Rare & Aug.-Sept. \\
\hline 115. & Holarrhena antidysenterica (L.) Wall & $\mathrm{T}$ & Apocynaceae & Kurchi & Common & May.-Aug. \\
\hline
\end{tabular}




\begin{tabular}{|c|c|c|c|c|c|c|}
\hline 116. & Holoptelea integrifolia (Roxb.)Planch. & $\mathrm{T}$ & Ulmaceae & Chhalla & Not common & Feb.-Mar. \\
\hline 117. & Hybanthus enneaspermus (L.) F.Muell. & $\mathrm{H}$ & Violaceae & Not known & Common & Whole year \\
\hline 118. & $\begin{array}{l}\text { Hygrophila salicifolia (Vahl)Nees in } \\
\text { Wall. }\end{array}$ & $\mathrm{S}$ & Acanthaceae & Not known & Not common & Aug.-Nov. \\
\hline 119. & $\begin{array}{l}\text { Hygrophila auriculata (Schumch.) } \\
\text { Heine }\end{array}$ & $\mathrm{S}$ & Acanthaceae & Kulekhara & Very common & Sept.-Feb. \\
\hline 120. & Hygrophila difformis (L.f.) Blume & $\mathrm{S}$ & Acanthaceae & Not known & Not common & Sep.-Feb. \\
\hline 121. & Hygrophila polysperma T.Anderson & $\mathrm{H}$ & Acanthaceae & Not known & Not common & Oct.-Feb. \\
\hline 122. & Hyptis suaveolens (L.) Ooit. & $\mathrm{S}$ & Lamiaceae & Bilati tulsi & Not common & Nov.-Apr. \\
\hline 123. & Ichnocarpus frutescens Naves. & $\mathrm{CL}$ & Apocynaceae & Shyamalata & Common & Aug.-Feb. \\
\hline 124. & Indigofera tinctoria $\mathrm{L}$. & $\mathrm{S}$ & Fabaceae & nil & Rare & Jan.-Mar. \\
\hline 125. & Ipomoea obscura Guill. & $\mathrm{H}$ & Convolvulaceae & Not known & Common & Sep.-Dec. \\
\hline 126. & Ixora arborea Lodd. & $\mathrm{H}$ & Rubiaceae & Not known & Common & Feb-Mar. \\
\hline 127. & Jatropha curcas L. & $\mathrm{T}$ & Euphorbiaceae & Bharenda & Not common & Mar.-May. \\
\hline 128. & Jatropha gossypifolia $\mathrm{L}$. & $\mathrm{S}$ & Euphorbiaceae & Lal-bharenda & Not common & Jul.-Sep.. \\
\hline 129. & Justicia diffusa Willd. & $\mathrm{H}$ & Acanthaceae & Not known & Common & Jul.-Feb. \\
\hline 130. & Justicia gendarussa Burm.f. & $\mathrm{S}$ & Acanthaceae & Jagat madan & Not common & Mar.-May. \\
\hline 131. & Lagerstroemia speciosa Pers. & $\mathrm{T}$ & Lythraceae & Jarul & Common & May.-Sep. \\
\hline 132. & Lannea coromandelica (Houtt.)Merr. & $\mathrm{T}$ & Anacardiaceae & Jiol & Less common & Dec.-Apr. \\
\hline 133. & Lantana camara $(\mathrm{L})$. & $\mathrm{S}$ & Verbenaceae & Not known & Common & Whole year \\
\hline 134. & Leonotis nepetifolia Schimp.ex Benth. & S & Lamiaceae & Not known & Not common & Oct.-Feb. \\
\hline 135. & Leucas cephalotes Sperng. & $\mathrm{H}$ & Lamiaceae & Not known & Common & Sep.-Dec. \\
\hline 136. & Lindenbergia macrostachya Benth. & $\mathrm{H}$ & Scrophulariaceae & Basanti & Not common & Aug.-Jan. \\
\hline 137. & Lindernia antipoda (L.) Alston & $\mathrm{H}$ & Scrophulariaceae & Not known & Not common & Aug.-Mar. \\
\hline 138. & Lindernia ciliata (Colsm.) Pennell. & $\mathrm{H}$ & Scrophulariaceae & Not known & Common & Aug.-Dec. \\
\hline 139. & Lindernia crustacea (L.) F.Muell. & $\mathrm{H}$ & Scrophulariaceae & Not known & Common & Jun.-Jan. \\
\hline 140. & Lindernia oppositifolia (L.) Mukherjee & $\mathrm{H}$ & Scrophulariaceae & Not known & Common & Sep.-Oct. \\
\hline 141. & Lippia geminata Kunth & $\mathrm{H}$ & Verbenaceae & Not known & Not common & Mar.-Dec. \\
\hline 142. & Ludwigia adscendens (L.) H.Hara & $\mathrm{H}$ & Onagaraceae & Kesardam & Common & Sep.-Jan. \\
\hline 143. & Madhuca indica & $\mathrm{T}$ & Sapotaceae & Mahua & Common & Mar.-Jun. \\
\hline 144. & Martynia annua $($ L. $)$ & $\mathrm{S}$ & Martyniaceae & Bagh nokh & Less Common & Aug.-Oct. \\
\hline 145. & Mazus pumilus (Burm.f.) & $\mathrm{H}$ & Scrophulariaceae & Not known & Less common & Sept.-Feb. \\
\hline 146. & Mecardonia procumbens Small. & $\mathrm{H}$ & Scrophulariaceae & Not known & Not common & Feb.-Apr. \\
\hline 147. & Melochia corchorifolia Wall.. & $\mathrm{H}$ & Sterculiaceae & Bon-pat & Common & May-Oct. \\
\hline 148. & Merremia tridentata (L.) Hallier f. & $\mathrm{H}$ & Convolvulaceae & Not known & Less common & Jul.-Feb. \\
\hline 149. & Meyna spinosa Roxb.ex.Link. & $\mathrm{S}$ & Rubiaceae & Moinakanta & Common & Mar.-May. \\
\hline 150. & Micrococca mercurialis Benth. & $\mathrm{H}$ & Euphorbiaceae & Not known & Not common & Dec.-Feb. \\
\hline 151. & Mikania micrantha Kunth. & $\mathrm{CL}$ & Asteraceae & Not known & Rare & Dec.-Jan. \\
\hline 152. & Mimosa pudica Mill. & $\mathrm{H}$ & Mimosaceae & Lajjaabati & Common & Aug.-Mar. \\
\hline 153. & Mitragyna Parvifolia Korth. & $\mathrm{T}$ & Rubiaceae & Keli kadam & Not common & May.-Jul. \\
\hline 154. & Mitrasacme alsinoides $\mathrm{R} . \mathrm{Br}$. & $\mathrm{H}$ & Loganiaceae & Not known & Common & Aug.-Oct. \\
\hline 155. & Mollugo pentaphylla $\mathrm{L}$. & $\mathrm{H}$ & Aizoaceae & Khet papra & Common & Sep.-Dec. \\
\hline 156. & Momordica dioica Wall. & $\mathrm{CL}$ & Cucurbitaceae & Bon karala & Common & Aug.-Oct. \\
\hline 157. & Morinda tomentosa B.Heyne . & $\mathrm{T}$ & Rubiaceae & Haridra & Less common & May.-Jan. \\
\hline
\end{tabular}




\begin{tabular}{|c|c|c|c|c|c|c|}
\hline 158. & Nicotiana plumbaginifolia Willd. & $\mathrm{H}$ & Solanaceae & Bon-tamak & Rare & Jun.-Oct. \\
\hline 159. & Ochna pumila Buch- Ham.ex D.Don & $\mathrm{T}$ & Ochnaceae & Makal & rare & Mar.-Jun. \\
\hline 160. & Ocimum americanum Auct,ex Benth. & $\mathrm{S}$ & Lamiaceae & Bantulshi & Not common & Aug.-Mar. \\
\hline 161. & Ocimum sanctum $\mathrm{L}$. & $\mathrm{S}$ & Lamiaceae & Tulshi & Common & Oct.-Feb. \\
\hline 162. & $\begin{array}{l}\text { Oldenlandia corymbosa Herb.Madr.ex } \\
\text { Wight.\&Arn. }\end{array}$ & $\mathrm{S}$ & Rubiaceae & Not known & Common & Sep.-Apr. \\
\hline 163. & Oxalis corniculata $\mathrm{L}$. & $\mathrm{H}$ & Oxalidaceae & Amrul & Very common & Oct-Jun. \\
\hline 164. & Paederia scandens (Lour.)Merr. & $\mathrm{T}$ & Rubiaceae & Gandalpata & Less common & Sep.-Nov. \\
\hline 165. & Pandanus fascicularis Lam. & $\mathrm{S}$ & Pandanaceae & Keya & Less common & Aug.-Oct. \\
\hline 166. & Passiflora foetida Vell. & $\mathrm{H}$ & Passifloraceae & Jhumkolata & Common & Jul.-Dec. \\
\hline 167. & Pavetta indica $\mathrm{L}$. & $\mathrm{CL}$ & Rubiaceae & Not known & Less common & Apr.-Aug. \\
\hline 168. & $\begin{array}{l}\text { Peltophorum pterocarpum (DC.) Baker } \\
\text { ex K.Heyne. }\end{array}$ & $\mathrm{T}$ & Caesalpiniaceae & Radhachura & Common & Jun.-Apr. \\
\hline 169. & Peperomia pellucida Kunth & $\mathrm{H}$ & Piperaceae & Not known & Not common & Oct.-Feb. \\
\hline 170. & Pergularia daemia (Forssk.) Chiov. & $\mathrm{H}$ & Asclepiadaceae & Chagalbati & Common & May.-July. \\
\hline 171. & Peristrophe bicalyculata Nees. & $\mathrm{H}$ & Acanthaceae & Not known & Not common & Sep.-Mar. \\
\hline 172. & Phoenix acaulis Buch-Ham.ex Roxb. & $\mathrm{T}$ & Arecaceae & Khejur & Common & Jan-May. \\
\hline 173. & Phyla nodiflora Greene & $\mathrm{H}$ & Verbenaceae & Not known & Less common & Aug.-Mar. \\
\hline 174. & Phyllanthus emblica L. & $\mathrm{T}$ & Euphorbiaceae & Amlaki & Not commo & Feb.-Jun. \\
\hline 175. & Phyllanthus fraternus G.L.Webster & $\mathrm{H}$ & Euphorbiaceae & Bhuin amla & Common & July.-Nov. \\
\hline 176. & Phyllanthus reticulatus Lodd. & $\mathrm{S}$ & Euphorbiaceae & Panjuli & Not common & Sep.-Oct, \\
\hline 177. & Physalis minima $\mathrm{L}$. & $\mathrm{H}$ & Solanaceae & Not known & Less common & Aug.-Jan. \\
\hline 178. & Plumbago zeylanica L. & $\mathrm{H}$ & Plumbaginaceae & Chitrak & Not common & Aug.-Sep. \\
\hline 179. & Polygala chinensis $\mathrm{L}$. & $\mathrm{H}$ & Polygalaceae & Not known & Common & Oct.-Feb. \\
\hline 180. & Polygonum hydropiper L. & $\mathrm{H}$ & Polygonaceae & Panimarich & Common & May.-Dec. \\
\hline 181. & Polygonum plebeium $\mathrm{R} . \mathrm{Br}$. & $\mathrm{S}$ & Polygonaceae & Chemti sak & Less common & Jan.-Jun. \\
\hline 182. & Pongamia pinnata (L.)Pierre. & $\mathrm{T}$ & Fabaceae & Karanj & Less common & May.-Aug. \\
\hline 183. & Portulaca quadrifida L. & $\mathrm{H}$ & Portulacaceae & Chotanunia & Very common & Whole year \\
\hline 184. & Pouzolzia zeylanica (L.)Benn. & $\mathrm{H}$ & Urticaceae & Not known & Not common & Aug.-Nov. \\
\hline 185. & Pterocarpus marsupium Roxb. & $\mathrm{T}$ & Fabeceae & Pia sal & Less common & Mar.-Jun. \\
\hline 186. & Rauvolfia tetraphylla L. & $\mathrm{S}$ & Apocynaceae & Sarpagandha & Rare & Apr.-Oct. \\
\hline 187. & Ruellia tuberosa $\mathrm{L}$. & $\mathrm{H}$ & Acanthaceae & Chatpati & Common & Jul.-Dec. \\
\hline 188. & Rungia pectinata Nees. & $\mathrm{H}$ & Acanthaceae & Not known & Common & Aug.-Mar. \\
\hline 189. & Saccharum spontaneum L. & $\mathrm{H}$ & Poaceae & Kash & Common & Sept.-Dec \\
\hline 190. & Salmalia malabarica Schott.\& Endl. & $\mathrm{T}$ & Bombacaceae & Simul & Common & JAN-Mar. \\
\hline 191. & Sapindus emarginatus Hort.Alger. & $\mathrm{T}$ & Sapindaceae & Ritha & rare & Sep.-Dec. \\
\hline 192. & Scoparia dulcis L. & $\mathrm{H}$ & Scrophulariaceae & Bon dhane & Common & Jun.-Nov. \\
\hline 193. & $\begin{array}{l}\text { Senna siamea }(\text { Lam).H.S.Irwin\& } \\
\text { Bameby }\end{array}$ & $\mathrm{T}$ & Caesalpiniaceae & Minjiri & Common & Sep.-Mar. \\
\hline 194. & Senna alata $\mathrm{L}$. & $\mathrm{S}$ & Caesalpiniaceae & Dadmardan & Less common & Apr.-Mar. \\
\hline 195. & Senna occidentalis (L) Link. & $\mathrm{S}$ & Caesalpiniaceae & Kalkasunda & Common & Jul.Apr. \\
\hline 196. & Senna tora (L.) Roxb. & $\mathrm{S}$ & Caesalpiniaceae & Not known & Common & Sep.Nov. \\
\hline 197. & Setaria glauca (L.) P.Beauv. & $\mathrm{H}$ & Poaceae & Not known & Less common & Jul.-Nov. \\
\hline 198. & Setaria verticillata (L.)P.Beauv. & $\mathrm{H}$ & Poaceae & Dora-byara & Less common & Aug.-Nov. \\
\hline 199. & Shorea robusta C.F.Gaertn. & $\mathrm{T}$ & Dipterocarpaceae & Sal & Common & Feb.-Apr. \\
\hline
\end{tabular}




\begin{tabular}{|c|c|c|c|c|c|c|}
\hline 200. & Sida acuta Burm.f. & $\mathrm{S}$ & Malvaceae & kureta & Common & Aug.-Jan. \\
\hline 201. & Sida cordifolia $\mathrm{L}$. & $\mathrm{S}$ & Malvaceae & Swet-Berela & Common & Aug.-Nov. \\
\hline 202. & Sida rhombifolia L. & $\mathrm{S}$ & Malvaceae & Berela & Common & Oct.-Dec. \\
\hline 203. & Smilax macrophylla Griseb. & $\mathrm{CL}$ & Smilacaceae & Ramdaton & Common & Jul.-Oct. \\
\hline 204. & Solanum sisymbriifolium Lam. & $\mathrm{S}$ & Solanaceae & Sad kantikari & Common & July- Oct \\
\hline 205. & Solanum surattense Burm. & $\mathrm{S}$ & Solanaceae & kantikari & Less common & Whole year \\
\hline 206. & Solanum torvum Buch.-Ham.ex Wall. & $\mathrm{S}$ & Solanaceae & Titabegun & Rare & Dec.-Apr. \\
\hline 207. & Sporobolus diander P.Beauv. & $\mathrm{H}$ & Poaceae & Bena-joni & Common & Jul.-Nov. \\
\hline 208. & Streblus asper Lour. & $\mathrm{T}$ & Moraceae & Saora & Common & Jan.-May, \\
\hline 209. & Striga angustifolia (D.Don) Saldanha & $\mathrm{H}$ & Scrophulariaceae & Not known & Rare & Sep.-Dec. \\
\hline 210. & Suregada multiflora Baill. & $\mathrm{T}$ & Euphorbiaceae & Not known & Not common & Mar.-May \\
\hline 211. & Tectona grandis L.f & $\mathrm{S}$ & Verbenaceae & Shegun & Common & July.-Oct. \\
\hline 212. & $\begin{array}{l}\text { Terminalia arjuna (Roxb.ex } \\
\text { DC)Wight.\& Arn. }\end{array}$ & $\mathrm{T}$ & Combretaceae & Arjun & Common & Apr.-Oct. \\
\hline 213. & Terminalia bellirica (Gaertn.) Roxb. & $\mathrm{T}$ & Combretaceae & Bahera & Rare & Mar.-Jan. \\
\hline 214. & Terminalia chebula Retz. & $\mathrm{T}$ & Combretaceae & Haritaki & Rare & Apr.-Dec. \\
\hline 215. & Tinospora cordifolia Miers & $\mathrm{CL}$ & Menispermaceae & Gulancha-lata & Very common & Aug.-Dec. \\
\hline 216. & Tragia involucrata L. & $\mathrm{H}$ & Euphorbiaceae & Bichuti & Common & Jan.-Apr. \\
\hline 217. & Tridax procumbens $\mathrm{L}$. & $\mathrm{H}$ & Asteraceae & Not known & Common & Whole year \\
\hline 218. & Triumfetta rhomboidea Lindl. & $\mathrm{S}$ & Tiliaceae & Bon okhra & common & Aug.-Nov. \\
\hline 219. & Turnera ulmifolia Sesse \& Moc. & $\mathrm{S}$ & Turneraceae & Not known & Common & Aug-Dec \\
\hline 220. & Uraria picta (Jacq.)Desv. & $\mathrm{H}$ & Fabaceae & Sibjata & Less common & Jul.-Oct \\
\hline 221. & Urena lobata $\mathrm{L}$. & $\mathrm{S}$ & Malvaceae & Ban-bhenda & Common & Sep.-Dec. \\
\hline 222. & Utricularia stellaris L.f. & $\mathrm{H}$ & Lentibulariaceae & Not known & Less common & Oct.-Dec. \\
\hline 223. & Vernonia cinerea (L.) Less. & $\mathrm{H}$ & Asteraceae & Not known & Common & Whole year \\
\hline 224. & Wahlenbergia gracilis (G.Forst.) A.DC. & $\mathrm{H}$ & Campanulaceae & Not known & Common & Whole year \\
\hline 225. & Wedelia chinensis Merr. & $\mathrm{H}$ & Asteraceae & Not known & Not common & Apr.-Oct. \\
\hline 226. & Zingiber montanum Link ex A.Dietr. & $\mathrm{H}$ & Zingiberaceae & Bon Ada & Not common & Aug.-Sep. \\
\hline 227. & Ziziphus mauritiana Lam. & $\mathrm{H}$ & Rhamnaceae & Kul & Less common & Aug.-Oct. \\
\hline 228. & Ziziphus oenoplia (L.) Mill. & $\mathrm{T}$ & Rhamnaceae & Shiakul & Common & Aug.-Nov. \\
\hline 229. & Zornia gibbosa Span. & $\mathrm{H}$ & Fabaceae & Not known & Common & Aug.-Oct. \\
\hline
\end{tabular}

Abbreviation used : $\mathrm{H}=\mathrm{Herb}, \mathrm{S}=\mathrm{Shrub}, \mathrm{Cl}=\mathrm{Climber}, \mathrm{T}=$ Tree, Jan.= January, Feb.= February, Mar.= March, Apr.= April, Jun.=June, Jul.=July, Aug.=August, Sep.=September, Oct.=October, Nov. $=$ November, Dec. $=$ December. 


\section{Table.2 Folk medicinal importance of some plant species of Ganpur forest, Birbhum District}

\begin{tabular}{|c|c|c|c|c|}
\hline Sl. No. & $\begin{array}{l}\text { Scientific name of the } \\
\text { plants }\end{array}$ & Ailments & Parts Used & Mode of use \\
\hline 1. & Abrus precatorius $\mathrm{L}$. & $\begin{array}{l}\text { Cough, cold, collie pains, } \\
\text { anti-tumour }\end{array}$ & Root, leaf, seed & $\begin{array}{l}\text { Root and leaf decoction is act as diuretic, seed } \\
\text { extract by boiling is used in nervous disorder and } \\
\text { antitumor }\end{array}$ \\
\hline 2. & Abutilon indicum (L.) Sweet & $\begin{array}{l}\text { demulcent, laxative, diuretic, } \\
\text { sedative, intestinal worm } \\
\text { infestation, }\end{array}$ & Whole plant & Leaf and fruit is taken orally. \\
\hline 3. & Acacia nilotica Dellile. & dysentery, & bark, leaves, & Bark-juice \& leaf-juice is eaten. \\
\hline 4. & Achyranthes aspera $\mathrm{L}$. & $\begin{array}{l}\text { Insect poisoning, diarrhoea, } \\
\text { diuretic }\end{array}$ & $\begin{array}{l}\text { Leaves paste, root } \\
\text { decoction }\end{array}$ & $\begin{array}{l}\text { Leaves paste are applied to the bite place and } \\
\text { decoction of root is given to the diarrhoea patient. }\end{array}$ \\
\hline 5. & Aegle marmelos (L.) Correa. & $\begin{array}{l}\text { Chronic dysentery, } \\
\text { constipation, dyspepsia, } \\
\text { diabetes }\end{array}$ & Fruit, leaf & $\begin{array}{l}\text { Ripe fruit is eaten or un-ripe fruit also been boiled } \\
\text { and eaten. Tender leaf juice about } 10 \mathrm{ml} \text { mixed } \\
\text { with } 2-3 \text { drops of honey is eaten in empty stomach } \\
\text { to control diabetes. }\end{array}$ \\
\hline 6. & $\begin{array}{l}\text { Aerva lanata (L.) Juss.ex } \\
\text { Schult. }\end{array}$ & Asthma & Flower with leaf juice & $5 \mathrm{ml}$ juice with few drops of honey for one month \\
\hline 7. & Agave americana $\mathrm{L}$. & diuretic & root & root-juice is eaten \\
\hline 8. & $\begin{array}{l}\text { Alangium salviifolium (L.f.) } \\
\text { Wangerin }\end{array}$ & Hydrophobia & Root & $\begin{array}{l}50 \text { gm root crushed are administered on the wound } \\
\text { made by dog bite }\end{array}$ \\
\hline 9. & Albizia lebbeck (L.) Benth. & Arthritis & Seed & Seed-paste is used on the affected site. \\
\hline 10. & $\begin{array}{l}\text { Alstonia scholaris (L.) } \\
\text { R.Br. }\end{array}$ & $\begin{array}{l}\text { Diarrhea, dysentery, ulcers, } \\
\text { sores, rheumatic inflation }\end{array}$ & Bark, latex & $\begin{array}{l}\text { Dried bark is taken for diarrhea, dysentery and } \\
\text { latex is used in ulcers, sores and rheumatic } \\
\text { inflation. }\end{array}$ \\
\hline 11. & $\begin{array}{l}\text { Alysicarpus monilifer (L.) } \\
\text { DC. }\end{array}$ & Inflammation, pain, jaundice & Leaf, root & $\begin{array}{l}\text { Leaf extract is used as pain reliever, root extract is } \\
\text { used for jaundice and pain. }\end{array}$ \\
\hline 12. & Amaranthus spinosus.L. & Diuretic, abscess, buboes & Root, whole plant & $\begin{array}{l}\text { Root crushed are used as diuretic and juice of the } \\
\text { whole plant is used in buboes and abscess }\end{array}$ \\
\hline 13. & Anacardium occidentale $\mathrm{L}$. & $\begin{array}{l}\text { Influenza, diarrhoea, Blood } \\
\text { pressure }\end{array}$ & Fruit, bark, seed & $\begin{array}{l}\text { Fruit juice is used for influenza, bark decoction is } \\
\text { used for controlling diarrhoea, seed is eaten for } \\
\text { controlling blood pressure }\end{array}$ \\
\hline 14. & $\begin{array}{l}\text { Andrographis paniculata } \\
\text { Nees. }\end{array}$ & Dyspepsia, liver trouble & Whole plants & $\begin{array}{l}\text { one dried small pill made from leaves paste taken } \\
\text { in empty stomach }\end{array}$ \\
\hline 15. & Annona reticulata Vell. & $\begin{array}{l}\text { Anthelmintic Anti- } \\
\text { dysenteric, Astringent }\end{array}$ & Fruit, bark & Unripe fruit are eaten, bark-juice is eaten \\
\hline 16. & $\begin{array}{l}\text { Argyreia nervosa } \\
\text { (Burm.f.)Bojer }\end{array}$ & Nervous disorder, eczema & Root, leaf & $\begin{array}{l}\text { Root is boiled in water and taken a cup of } \\
\text { decoction, leaf-paste is applied on eczema. }\end{array}$ \\
\hline 17. & Aristolochia indica $\mathrm{L}$. & $\begin{array}{l}\text { Snake bite, food poisoning in } \\
\text { cows }\end{array}$ & Bark, root juice & one cup juice daily till cure \\
\hline 18. & $\begin{array}{l}\text { Asparagus racemosus } \\
\text { Willd. }\end{array}$ & $\begin{array}{l}\text { Anti-diarrhoea, diuretic, anti- } \\
\text { dysenteric, nutritive }\end{array}$ & Root & Juice of fasciculate roots or boiled roots are eaten. \\
\hline 19. & Azadirachta indica A.Juss. & $\begin{array}{l}\text { Antiseptic, blood purifier, } \\
\text { small pox, }\end{array}$ & $\begin{array}{l}\text { Leaf, seed, bark, } \\
\text { flower }\end{array}$ & $\begin{array}{l}\text { Leaf-juice } 1 \text { tea spoon is eaten or plants are eaten } \\
\text { by cooking for blood purifier.oil from seed is used } \\
\text { as antiseptic .leaf-paste is used in small pox }\end{array}$ \\
\hline 20. & Barleria cristata Lam. & Cough, swellings & Leaves, roots & $\begin{array}{l}\text { Leaf juice } 2 \text { tea spoon are taken, Root paste is } \\
\text { applied on the swelling site. }\end{array}$ \\
\hline 21. & Barleria prionitis L. & $\begin{array}{l}\text { Fever, respiratory disease, } \\
\text { tooth ache }\end{array}$ & Leaves and bark & $\begin{array}{l}\text { Leaf juice are taken for fever and respiratory } \\
\text { disease, bark is used to for curing tooth ache }\end{array}$ \\
\hline 22. & Bixa orellana $\mathrm{L}$. & $\begin{array}{l}\text { Purgative, astringent, } \\
\text { jaundice }\end{array}$ & Fruit, seed & $\begin{array}{l}\text { fruit is eaten as purgative and astringent, leave } \\
\text { juice is taken to cure jaundice }\end{array}$ \\
\hline 23. & $\begin{array}{l}\text { Boerhavia diffusa } \\
\text { Engehm.\& A.Gray }\end{array}$ & $\begin{array}{l}\text { Laxative, diuretic, } \\
\text { expectorant, anaemia, } \\
\text { jaundice, skin diseases }\end{array}$ & Whole plant & Decoction of plant $1-2$ tea spoonful is eaten. \\
\hline 24. & Bridelia retusa Spreng. & $\begin{array}{l}\text { Astringent, causing } \\
\text { infertility }\end{array}$ & Root, bark & Root pest are taken every alternate day. \\
\hline
\end{tabular}




\begin{tabular}{|c|c|c|c|c|}
\hline 25. & $\begin{array}{l}\text { Bryophyllum calycinum } \\
\text { Salisb. }\end{array}$ & $\begin{array}{l}\text { Kidney \& urinary bladder } \\
\text { stone, cuts }\end{array}$ & Plant Juice. Leaf & $\begin{array}{l}\text { Plant-juice is eaten till the stone is cleared; leaf- } \\
\text { juice is externally use in cuts. }\end{array}$ \\
\hline 26. & Buchanania lanzan Spreng. & Wound & Root & Root extract is used for wound healing, \\
\hline 27. & $\begin{array}{l}\text { Butea monosperma (Lam.) } \\
\text { Taub. }\end{array}$ & $\begin{array}{l}\text { Anti-fertility, piles, } \\
\text { diarrhoea, dysentery, diuretic }\end{array}$ & $\begin{array}{l}\text { Seed, flower, gum, } \\
\text { bark }\end{array}$ & $\begin{array}{l}\text { Seeds and flowers are used as ant fertility, gum is } \\
\text { used to control diarrhoea, bark is used in piles and } \\
\text { tumours. }\end{array}$ \\
\hline 28. & $\begin{array}{l}\text { Butea superba } \\
\text { Roxb.ex.Wild. }\end{array}$ & Weakness & Root & $\begin{array}{l}\text { Root extract is used for mental and physical } \\
\text { weakness }\end{array}$ \\
\hline 29. & $\begin{array}{l}\text { Cardiospermum } \\
\text { halicacabum } \mathrm{L} .\end{array}$ & $\begin{array}{l}\text { Rheumatism, diuretic, nerve } \\
\text { diseases }\end{array}$ & Root & Root-juice is eaten. \\
\hline 30. & $\begin{array}{l}\text { Carissa spinarum } \\
\text { Lodd.ex.A.DC. }\end{array}$ & Purgative & Root & Root-paste are eaten. \\
\hline 31. & Cassia fistula $\mathrm{L}$. & Artritis & Fruit & $\begin{array}{l}\text { One table spoon powder of fruit is taken orally for } \\
7 \text { days. }\end{array}$ \\
\hline 32. & Centella asiatica Urb. & $\begin{array}{l}\text { Diuretic,blood } \\
\text { purifier,leprosy }\end{array}$ & Leaf,stem & $\begin{array}{l}\text { Leaves and stem juice is taken in the empty } \\
\text { stomach } 5 \text { days at asteach. }\end{array}$ \\
\hline 33. & Cissus quadrangularis L. & $\begin{array}{l}\text { Setting fractured bone, } \\
\text { anthelmintic, blood purifier, } \\
\text { scurvy }\end{array}$ & Stem, root & $\begin{array}{l}\text { Stem and root paste is used in setting fractured } \\
\text { bones, stem juice is used as anthelmintic, blood } \\
\text { purifier and in scurvy. }\end{array}$ \\
\hline 34. & Cleome viscosa $\mathrm{L}$. & chronic rheumatism, ear ache & Seed paste, leaf juice & $\begin{array}{l}\text { seed paste are applied on the joint pain, leaf juice } \\
\text { is taken for earache }\end{array}$ \\
\hline 35. & $\begin{array}{l}\text { Corchorus aestuans } \\
\text { Herb.Madr.ex Wall. }\end{array}$ & $\begin{array}{l}\text { Anaemia, pre delivery } \\
\text { trouble of Pregnant women. }\end{array}$ & Leaf, root. & $\begin{array}{l}\text { Leaves' decoction used for treating anaemia; root } \\
\text { extract mixed with that of Sida rhombifolia root is } \\
\text { taken once daily by pregnant women to relieve } \\
\text { from pre delivery trouble. }\end{array}$ \\
\hline 36. & $\begin{array}{l}\text { Corchorus fascicularis } \\
\text { Lam. }\end{array}$ & Ulcer in mouth, impotence & Whole plant & $\begin{array}{l}\text { Whole plant is dried and made powder and taken } \\
\text { orally }\end{array}$ \\
\hline 37. & $\begin{array}{l}\text { Costus speciosus (J.Koenig } \\
\text {.) Sm. }\end{array}$ & Purgative, anthelmintc. & Rhizome, root & $\begin{array}{l}\text { Rhizomes are cooked and eaten as purgative, roots } \\
\text { are used as anthelmintic. }\end{array}$ \\
\hline 38. & $\begin{array}{l}\text { Crotalaria prostrata } \\
\text { Rottler. }\end{array}$ & Rheumatism, & Seeds & Seeds are eaten within a banana \\
\hline 39. & Crotalaria verrucosa $\mathrm{L}$. & Skin allergies, Rheumatism & Leaf & Leaf extract is applied to the affected areas \\
\hline 40. & $\begin{array}{l}\text { Curculigo orchioides } \\
\text { Gaertn. }\end{array}$ & $\begin{array}{l}\text { vitality, vigour and Strength } \\
\text {, anticancer }\end{array}$ & Root & Root decoction is taken for one month. \\
\hline 41. & Curcuma aromatica Salisb. & Sprains & Rhizomes & Rhizomes paste is used in sprain \\
\hline 42. & Cuscuta reflexa Roxb. & Loose motion in cows & Stem juice & Fed to the cows thrice daily till cure \\
\hline 43. & $\begin{array}{l}\text { Dendrophthoe falcata } \\
\text { Blume }\end{array}$ & $\begin{array}{l}\text { Wounds, skin diseases, } \\
\text { antitumor potential }\end{array}$ & Arial part, leaf & Paste is applied in the affected site \\
\hline 44. & $\begin{array}{l}\text { Desmodium gangeticum } \\
\text { (L.) DC. }\end{array}$ & Anti-tumour & Fruit & Fruit paste applied on tumour \\
\hline 45 . & $\begin{array}{l}\text { Desmodium triflorum (L.) } \\
\text { DC. }\end{array}$ & Epilepsy & Root & Root extract is given orally \\
\hline 46. & Dillenia pentagyna Roxb. & antitumor & bark & Bark juice is taken with honey. \\
\hline 47. & Dioscorea alata $\mathrm{L}$. & $\begin{array}{l}\text { Ulcers, piles, dysentery, } \\
\text { syphilis }\end{array}$ & Tubers, leaves & $\begin{array}{l}\text { Tubers are taken raw and the leaves pests are used } \\
\text { to prevent the disease. }\end{array}$ \\
\hline 48. & Dioscorea bulbifera $\mathrm{L}$. & Ulcer, piles, dysentery & Tuber & $\begin{array}{l}\text { Dried tubers are powdered and applied on ulcer, } \\
\text { also taken to cure dysentery and piles. }\end{array}$ \\
\hline 49. & Drosera burmanni DC. & Rubefacient action & whole plant & Paste is applied in the affected site \\
\hline 50. & Eclipta prostrata (L.) & $\begin{array}{l}\text { Hair tonic, tooth-ache, } \\
\text { jaundice, purgative, spleen } \\
\text { enlargement }\end{array}$ & Leaf, plant & $\begin{array}{l}\text { Leaf-juice is used as black hair- dye , plant-juice } \\
\text { is used in spleen disease, purgative . }\end{array}$ \\
\hline 51. & Eupatorium odoratum $\mathrm{L}$. & Wounds, cuts & Leaf & Leaf-paste is used in the affected area. \\
\hline 52. & Ficus benghalensis L. & $\begin{array}{l}\text { Diarrhoea, dysentery, } \\
\text { diabetes, rheumatism }\end{array}$ & Bark, latex & $\begin{array}{l}\text { Infushion of bark is used for Diarrhoea, dysentery, } \\
\text { diabetes. latex is used for rheumatism }\end{array}$ \\
\hline
\end{tabular}




\begin{tabular}{|c|c|c|c|c|}
\hline 53. & $\begin{array}{l}\text { Flacourtia jangomas } \\
\text { (Lour.) Raeusch. }\end{array}$ & $\begin{array}{l}\text { Diuretic, digestive, } \\
\text { appetizer, jaundice, spleen } \\
\text { enlargement, urine problem }\end{array}$ & Fruit, bark, root & $\begin{array}{l}\text { Fruit is eaten as Diuretic, digestive, appetizer; } \\
\text { bark is used for spleen enlargement; root juice is } \\
\text { used for urine clear. }\end{array}$ \\
\hline 54. & $\begin{array}{l}\text { Flacourtia indica } \\
\text { (Burm.f.)Merr. }\end{array}$ & Wasp bite & stem \& bark paste & Paste is applied in the wound \\
\hline 55. & Gloriosa superba $\mathrm{L}$. & $\begin{array}{l}\text { Purgative, anthelmintic, } \\
\text { leprosy, piles, gonorrhoea, } \\
\text { gout }\end{array}$ & Root, leaf & $\begin{array}{l}\text { Tuber paste is used in leprosy, gonorrhoea } \\
\text { purgative, leaf-juice in piles and use to kill lice in } \\
\text { hair. }\end{array}$ \\
\hline 56. & Grewia hirsute Roxb. & Dysentery & Leaf & Leaf-decoction is eaten \\
\hline 57. & $\begin{array}{l}\text { Gymnema sylvestre (Retz.) } \\
\text { R.Br.ex Sm }\end{array}$ & $\begin{array}{l}\text { Diabetes, expectorant, } \\
\text { increasing urine }\end{array}$ & Leaf, root & $5 \mathrm{ml}$ leaf-juice daily for one month \\
\hline 58. & Heliotropium indicum $\mathrm{L}$. & $\begin{array}{l}\text { Ulcer wound, urticaria, } \\
\text { cough, fever }\end{array}$ & Leaf, root & $\begin{array}{l}\text { Leaf-paste are applied locally, leaf-decoction is } \\
\text { used in urticaria, root decoction is used in fever } \\
\text { and cough. }\end{array}$ \\
\hline 59. & $\begin{array}{l}\text { Hemidesmus indicus }(\mathrm{L} .) \\
\text { R.Br. }\end{array}$ & $\begin{array}{l}\text { Blood purifier, fever, } \\
\text { demulcent, diuretic, skin } \\
\text { diseases, syphilis etc }\end{array}$ & Roots & $\begin{array}{l}\text { Roots are put into water overnight and the } \\
\text { decoction taken } 1 \text { cup daily for a month or root } \\
\text { paste } 1 \text { tea spoon can be taken for a week./rubbed } \\
\text { on skin }\end{array}$ \\
\hline 60. & $\begin{array}{l}\text { Hemigraphis hirta } \\
\text { T.Anderson }\end{array}$ & Jaundice & Whole Plant & $\begin{array}{l}\text { Whole Plant with banana root is made a paste and } \\
\text { eaten thrice a day for a month. }\end{array}$ \\
\hline 61. & $\begin{array}{l}\text { Holarrhena antidysenterica } \\
\text { (L.) Wall }\end{array}$ & Dysentery & Bark & Bark-juice is eaten. \\
\hline 62. & $\begin{array}{l}\text { Hybanthus enneaspermus } \\
\text { (L.) F.Muell. }\end{array}$ & Diuretic, demulcent & Leaf, Root & Decoction of leaf and root is used for the puposes. \\
\hline 63. & $\begin{array}{l}\text { Hygrophila auriculata } \\
\text { (Schumch.) Heine }\end{array}$ & Diuretic, jaundice, dropsy & whole plant & Leaf-juice is eaten or plants are eaten by cooking. \\
\hline 64. & Hyptis suaveolens (L.) Ooit. & Itching, cough, cold & Leaf & Leaf-juice is used \\
\hline 65. & $\begin{array}{l}\text { Ichnocarpus frutescens } \\
\text { Naves. }\end{array}$ & $\begin{array}{l}\text { Diuretic, diabetes, bladder } \\
\text { stone }\end{array}$ & Root & Dried root powder is used one tea spoon daily \\
\hline 66. & Indigofera tinctoria $\mathrm{L}$. & Epilepsy, hydrophobia & Leaf & $\begin{array}{l}\text { Leaf extract is used in epilepsy, leaf juice is used } \\
\text { in hydrophobia. }\end{array}$ \\
\hline 67. & Jatropha curcas $\mathrm{L}$. & wounds, cut, toothache etc. & Latex & Latex smeared on the wound, cut, tooth-ache \\
\hline 68. & Jatropha gossypifolia $\mathrm{L}$. & Leprosy, gum-ache & Root, shoot & $\begin{array}{l}\text { Roots are made paste and externally apply, shoot } \\
\text { extract is used in gum }\end{array}$ \\
\hline 69. & $\begin{array}{l}\text { Lannea coromandelica } \\
\text { (Houtt.)Merr. }\end{array}$ & Skin disease, ulcer, sprain & $\begin{array}{l}\text { Bark, Tender leaf and } \\
\text { root }\end{array}$ & $\begin{array}{l}\text { Bark is used for skin disease, Tender leaf and root } \\
\text { is used for sprain and ulcer. }\end{array}$ \\
\hline 70. & $\begin{array}{l}\text { Leonotis nepetifolia } \\
\text { Schimp.ex Benth. }\end{array}$ & Burns & Seed & Seed is made paste and applied on the burn site. \\
\hline 71. & Martynia annua (L.) & Epilepsy & Leaf, fruit & $\begin{array}{l}\text { Two spoon of leaf-juice or fruit-juice is eaten for } \\
\text { one week. }\end{array}$ \\
\hline 72. & $\begin{array}{l}\text { Melochia corchorifolia } \\
\text { Wall.. }\end{array}$ & Dysentery & Leaf & Leaf-decoction is taken \\
\hline 73. & Mimosa pudica Mill. & Infertility & root juice & 1 tea spoon juice with pepper for one month \\
\hline 74. & $\begin{array}{l}\text { Ochna pumila Buch- } \\
\text { Ham.ex D.Don }\end{array}$ & $\begin{array}{l}\text { epilepsy, menstrual } \\
\text { complaints, asthma, }\end{array}$ & Leaf, flower, bark & $\begin{array}{l}\text { Flower is dried to make powder and use in } \\
\text { epilepsy, leaf and bark paste is used in menstrual } \\
\text { problem and asthma. }\end{array}$ \\
\hline 75 . & $\begin{array}{l}\text { Ocimum americanum } \\
\text { Auct,ex Benth. }\end{array}$ & Cough, dysentery & Leaf & Decoction of leaves is used. \\
\hline 76. & Ocimum sanctum $\mathrm{L}$. & Cough, cold and diabetes & Leaf, inflorescence & $\begin{array}{l}\text { Leaves } 8-10 \text { with } 2 \text { inflorescence is boiled with } \\
\text { ginger and decoction is mixed with 1tea-spoon of } \\
\text { honey and eaten in empty stomach. }\end{array}$ \\
\hline 77. & Oxalis corniculata $\mathrm{L}$. & $\begin{array}{l}\text { Appetizer, headache, } \\
\text { dyspepsia, piles }\end{array}$ & Whole plant & Fresh juice of the plant is taken \\
\hline 78. & $\begin{array}{l}\text { Peristrophe bicalyculata } \\
\text { Nees. }\end{array}$ & $\begin{array}{l}\text { Tuberculostatic, antidote to } \\
\text { snake poison }\end{array}$ & Whole plant & Fresh Plant crushed are eaten with rice infusion \\
\hline 79. & Phyllanthus emblica L. & $\begin{array}{l}\text { Dyspepsia, purgative, scurvy } \\
\text {, hair tonic }\end{array}$ & Fruit & Raw fruits are eaten or dried fruits also eaten. \\
\hline
\end{tabular}




\begin{tabular}{|c|c|c|c|c|}
\hline 80. & $\begin{array}{l}\text { Phyllanthus fraternus } \\
\text { G.L.Webster }\end{array}$ & High bilirubin & plant juice & $\begin{array}{l}\text { 5ml juice once daily for } 10 \text { days in the empty } \\
\text { stomach }\end{array}$ \\
\hline 81. & Physalis minima $\mathrm{L}$. & $\begin{array}{l}\text { Diuretic, laxative, spleen } \\
\text { disorder }\end{array}$ & Fruit, leaf & $\begin{array}{l}\text { Ripe Fruit and leaf-paste is eaten as laxative and } \\
\text { diuretic. }\end{array}$ \\
\hline 82. & Plumbago zeylanica $\mathrm{L}$. & $\begin{array}{l}\text { Diuretic, bile secretion, } \\
\text { diarrhea, piles, dyspepsia }\end{array}$ & Root-bark & Raw root-bark is pasted and used. \\
\hline 83. & Polygala chinensis L. & Anti-inflammatory & Whole plant & Plant paste is taken orally \\
\hline 84. & Polygonum hydropiper L. & $\begin{array}{l}\text { Stimulant, diuretic, } \\
\text { haemostatic }\end{array}$ & Root, whole plant & $\begin{array}{l}\text { Root paste/dried root powder is eaten diuretic; } \\
\text { infusion of plant is used as stimulant, diuretic and } \\
\text { haemostatic. }\end{array}$ \\
\hline 85. & $\begin{array}{l}\text { Pongamia pinnata } \\
\text { (L.)Pierre. }\end{array}$ & Rheumatoid arthritis & Seed & $\begin{array}{l}\text { Oil is extracted from the seeds and applied on the } \\
\text { affected area. }\end{array}$ \\
\hline 86. & Portulaca quadrifida $\mathrm{L}$. & $\begin{array}{l}\text { Hepatic disorders, heart, } \\
\text { kidney and bladder diseases }\end{array}$ & whole plant & Plant is taken as vegetable diet to cure the diseases \\
\hline 87. & Rauvolfia tetraphylla L. & Snake bite, diabetes mellitus & Root juice & $\begin{array}{l}10 \mathrm{ml} \text { juice is given orally and also apply in the } \\
\text { wound }\end{array}$ \\
\hline 88. & $\begin{array}{l}\text { Salmalia malabarica } \\
\text { Schott.\& Endl. }\end{array}$ & $\begin{array}{l}\text { Gonorrhea,diuretic, } \\
\text { astringent }\end{array}$ & Root, bark, gum & Root decoction is used for the purposes. \\
\hline 89. & $\begin{array}{l}\text { Sapindus emarginatus } \\
\text { Hort.Alger. }\end{array}$ & $\begin{array}{l}\text { anti-inflammatory, } \\
\text { antipyretic }\end{array}$ & Seed, fruit & $\begin{array}{l}\text { Oil from seed is used as anti-inflammatory, Fruit } \\
\text { is used as antipyretic and hair problem. }\end{array}$ \\
\hline 90. & Senna alata $\mathrm{L}$. & Ringworm & Leaf & Leaf-paste is applied on ringworm. \\
\hline 91. & Senna occidentalis (L) Link. & $\begin{array}{l}\text { Purgative, skin diseases, } \\
\text { diuretic }\end{array}$ & Leaf, root, seed & $\begin{array}{l}\text { Leaf and root decoction is taken as purgative and } \\
\text { diuretic, seed paste is used for skin disease. }\end{array}$ \\
\hline 92. & Senna tora (L.) Roxb. & $\begin{array}{l}\text { Ringworm, eczema, } \\
\text { purgative }\end{array}$ & Leaf, seed & $\begin{array}{l}\text { Leaf and seed paste is applied on ringworm and } \\
\text { eczema, leaf paste also used as purgative. }\end{array}$ \\
\hline 93. & Sida acuta Burm.f. & $\begin{array}{l}\text { Diuretic, nerve disorder, } \\
\text { rheumatism }\end{array}$ & Leaves, Root & $\begin{array}{l}\text { Leaves juice is used for diuretic and mixed with } \\
\text { boil oil to make a paste and used in rheumatism, } \\
\text { root extract are used in nerve disease. }\end{array}$ \\
\hline 94. & Sida cordifolia $\mathrm{L}$. & Blood vomiting & root/leaf crushed & one cup of paste during vomiting \\
\hline 95. & Sida rhombifolia $L$. & Arthritis & Leaf-paste & Leaf-paste is applied on the joints. \\
\hline 96. & Smilax macrophylla Griseb. & $\begin{array}{l}\text { Blood dysentery, night } \\
\text { wetting }\end{array}$ & Root juice, leaf juice & $\begin{array}{l}1 / 2 \text { cup juice is taken daily till complete cure, } \\
\text { water kept in the leaf for overnight is drunk in } \\
\text { empty stomach to stop night wetting. }\end{array}$ \\
\hline 97. & $\begin{array}{l}\text { Solanum sisymbriifolium } \\
\text { Lam. }\end{array}$ & Gastric trouble & Fruit & Fruit juice is eaten \\
\hline 98. & Solanum surattense Burm. & $\begin{array}{l}\text { Chronic fever,scabies,skin } \\
\text { diseases prolong .mense } \\
\text { problem }\end{array}$ & Leaf, whole plant & Boil leaf in water and eaten $1 / 2$ cup daily \\
\hline 99. & $\begin{array}{l}\text { Solanum torvum Buch.- } \\
\text { Ham.ex Wall. }\end{array}$ & $\begin{array}{l}\text { Liver enlargement, diuretic, } \\
\text { expectorant, sedative } \\
\text {,toothache }\end{array}$ & Plant body, fruit, seed & $\begin{array}{l}\text { Plant juice is used as expectorant, diuretic } \\
\text {,sedative, fruit decoction is used in live } \\
\text { enlargement, cough and seed -fumes is inhaled for } \\
\text { curing toothache. }\end{array}$ \\
\hline 100. & $\begin{array}{l}\text { Terminalia arjuna (Roxb.ex } \\
\text { DC)Wight.\& Arn. }\end{array}$ & $\begin{array}{l}\text { hypertension ,cirrhosis of } \\
\text { liver }\end{array}$ & bark & dried bark is powdered and is used \\
\hline 101. & $\begin{array}{l}\text { Terminalia bellirica } \\
\text { (Gaertn.) Roxb. }\end{array}$ & $\begin{array}{l}\text { Astringent, diuretic, dropsy, } \\
\text { purgative }\end{array}$ & Fruits, bark & $\begin{array}{l}\text { Fruits are mixed with dried myrobalan and taken } \\
\text { as an astringent, purgative, bark juice is used for } \\
\text { diuretic }\end{array}$ \\
\hline 102. & Terminalia chebula Retz. & $\begin{array}{l}\text { Cough, } \\
\text { Laxative,ulcer,wounds, diuret } \\
\text { ic,cardio-tonic }\end{array}$ & Fruit & $\begin{array}{l}\text { Dried fruits are crushed and taken. Fruits are put } \\
\text { into } 200 \mathrm{ml} \text { water overnight and decoction is taken }\end{array}$ \\
\hline 103. & Tinospora cordifolia Miers & Fever, jaundice & leaves, stem & stem juice is given to high fever and jaundice \\
\hline 104. & Tragia involucrata $\mathrm{L}$. & $\begin{array}{l}\text { Spleen enlargement, guinea } \\
\text { worms }\end{array}$ & Fruit, root & $\begin{array}{l}\text { 2-3Fruit paste is eaten for spleen enlargement, } \\
\text { root paste is eaten one tea spoon for guinea worm }\end{array}$ \\
\hline 105. & $\begin{array}{l}\text { Triumfetta rhomboidea } \\
\text { Lindl. }\end{array}$ & Diabetes & Whole plant & Dried plants are taken orally. \\
\hline 106. & Uraria picta (Jacq.)Desv. & Hysteria & Leaf & Leaf paste is applied on whole body \\
\hline
\end{tabular}




\begin{tabular}{|r|l|l|l|l|}
\hline 107. & Urena lobata L. & Diuretic & root & Roots dried and crushed and used \\
\hline 108. & Vernonia cinerea (L.) Less. & Indigestion & Leaf & $\begin{array}{l}\text { Rhizomes is used } \\
\text { stimulant }\end{array}$ \\
\hline 109. & $\begin{array}{l}\text { Zingiber montanum Link ex } \\
\text { A.Dietr. }\end{array}$ & Diarrhoea, stimulant & Rhizomes & paste rubbed on abdomen \\
\hline 110. & Ziziphus mauritiana Lam. & Flatulence & leaf paste & $\begin{array}{l}\text { Decoction of bark is used for dysentery and uterus } \\
\text { infection }\end{array}$ \\
\hline 111. & Ziziphus oenoplia (L.) Mill. & Dysentery, uterus infection & Stem bark & Seed decoction is used forpreventing Dysentry \\
\hline 112. & Zornia gibbosa Span. & Dysentry, & Seed & \\
\hline
\end{tabular}

Table.3 Eleven dominant families of flowering plants of Ganpur forest:

\begin{tabular}{|l|l|l|l|}
\hline SI.No. & Family & Genera & Species \\
\hline 1 & Poaceae & 15 & 16 \\
\hline 2 & Fabaceae & 11 & 15 \\
\hline 3 & Acanthaceae & 9 & 14 \\
\hline 4 & Euphorbiaceae & 8 & 12 \\
\hline 5 & Asteraceae & 11 & 11 \\
\hline 6 & Rubiaceae & 11 & 11 \\
\hline 7 & Scrophulariaceae & 6 & 9 \\
\hline 8 & Caesalpiniaceae & 3 & 7 \\
\hline 9 & Lamiaceae & 5 & 6 \\
\hline 10 & Apocynaceae & 5 & 5 \\
\hline 11 & Verbenaceae & 5 & 5 \\
\hline \multicolumn{2}{|c|}{ TOTAL } & 89 & 111 \\
\hline
\end{tabular}

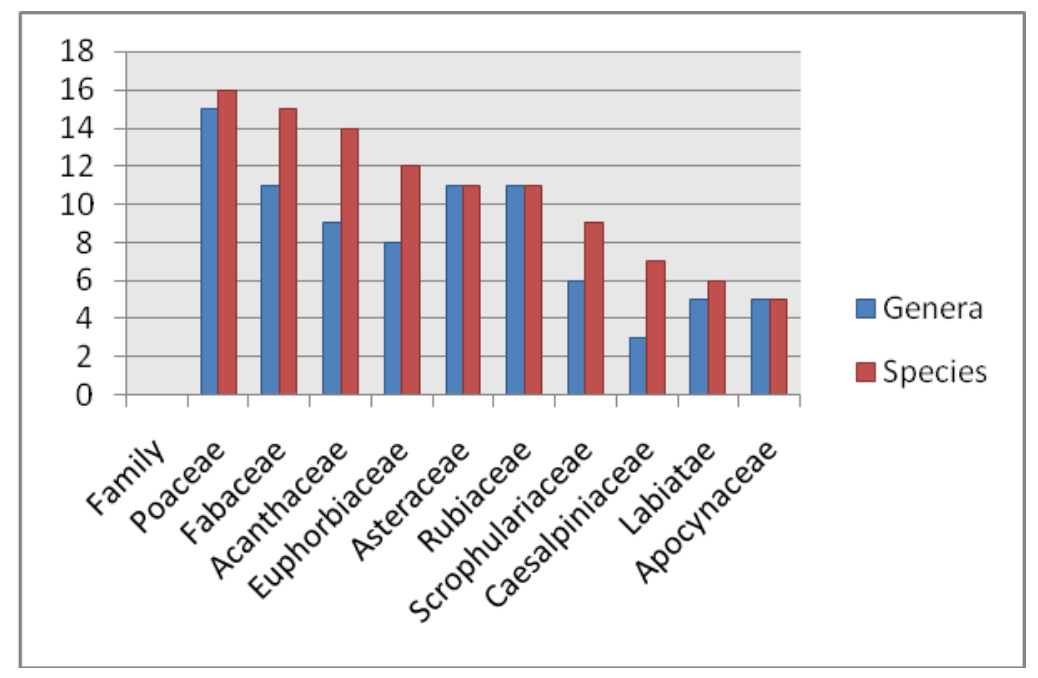

Figure.1 Ten dominant families of flowering plants 


\section{Photographs of plants and forest}

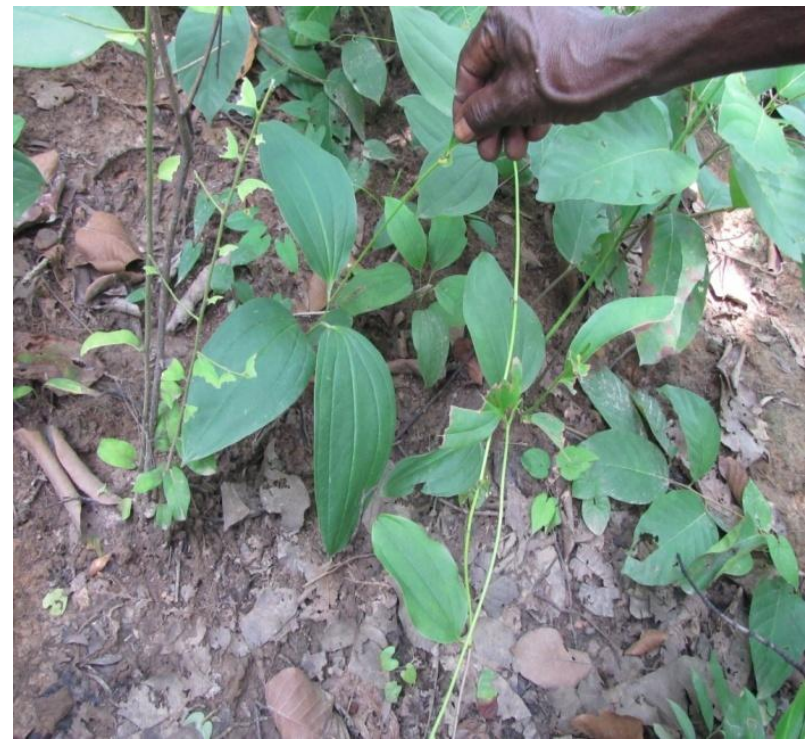

Smilax macrophylla (Smilacaceae)

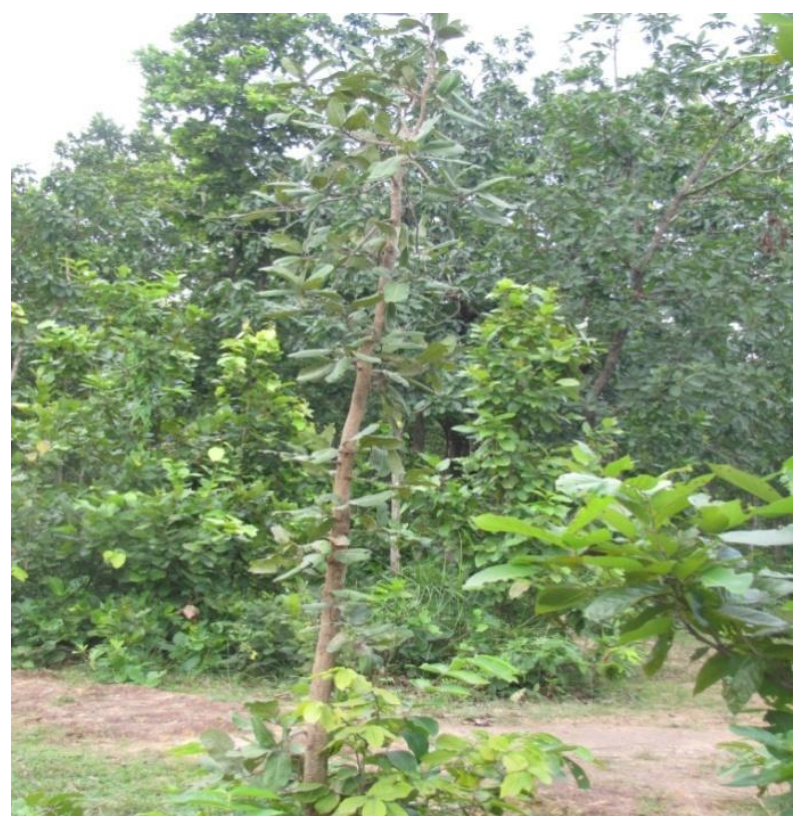

Buchanania lanzen (Anacardiaceae)

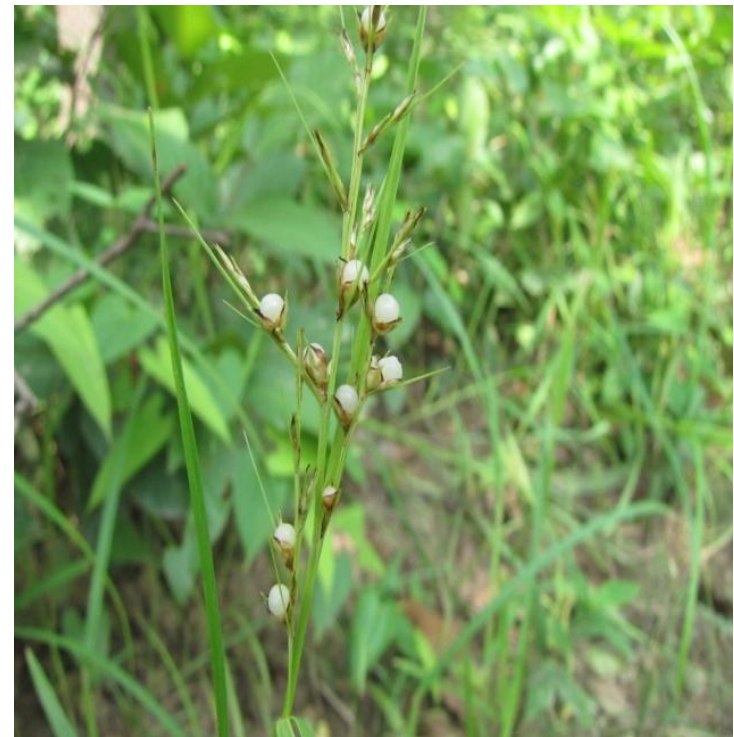

Coix lacryma-jobi (Poaceae)

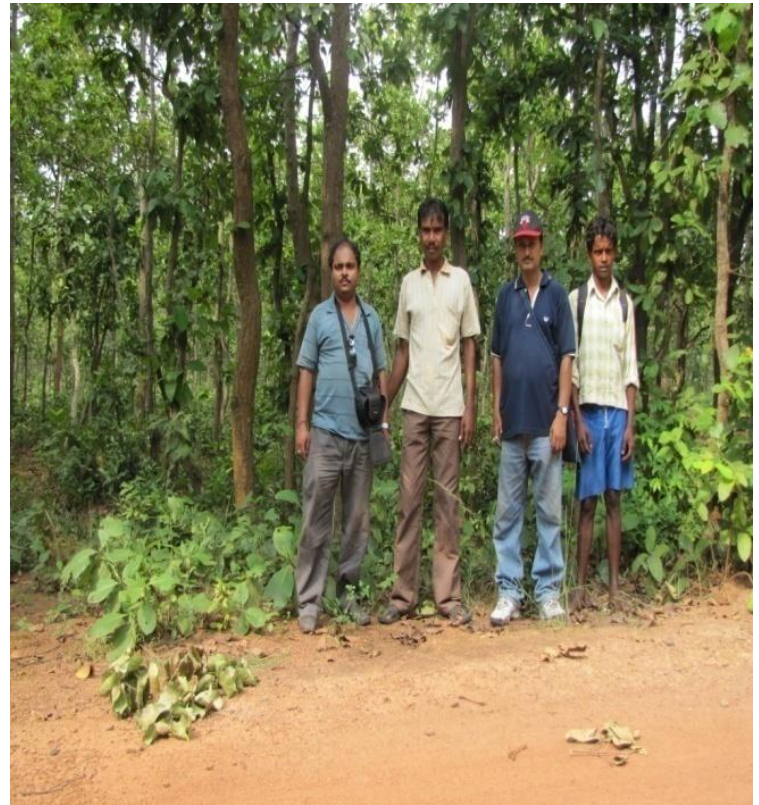

At Gonpur Forest 
Table.4 Life form composition of Ganpur forest

\begin{tabular}{|l|l|l|l|}
\hline Sl.No. & Lifeform & $\begin{array}{c}\text { Total Number } \\
\text { of Taxa }\end{array}$ & \% \\
\hline 1 & Herbs & 116 & 50.66 \\
\hline 2 & Shrubs & 43 & 18.34 \\
\hline 3 & Trees & 54 & 24.02 \\
\hline 4 & Climbers & 16 & 6.99 \\
\hline Total & & 229 & \\
\hline
\end{tabular}

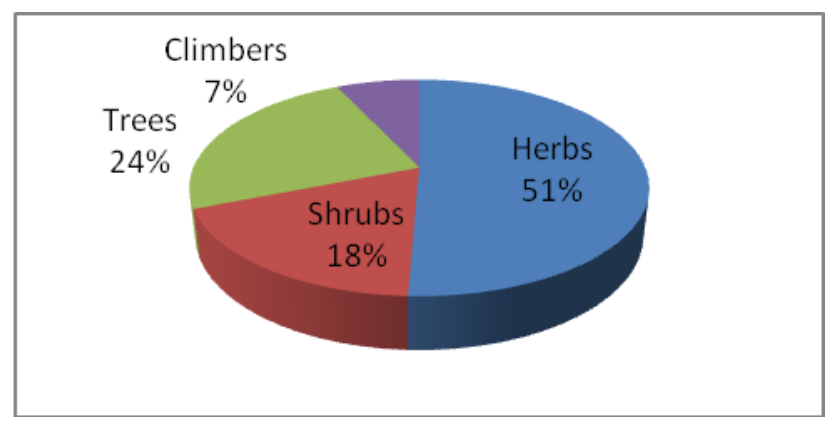

Figure.2 Life form composition of Ganpur Forest

Table.5 Statistics of the floristic composition of Ganpur forest

\begin{tabular}{|l|l|l|l|l|l|l|}
\hline \multirow{2}{*}{ Group } & \multicolumn{2}{|l|}{ Families } & \multicolumn{2}{l|}{ Genera } & \multicolumn{2}{l|}{ Species } \\
\cline { 2 - 7 } & No. & $\mathbf{\%}$ & No. & \% & No. & \% \\
\hline Dicotyledons & 70 & 86 & 154 & 84 & 193 & 84 \\
\hline Monocotyledons & 11 & 14 & 30 & 16 & 36 & 16 \\
\hline
\end{tabular}

Among these the following plant species are gradually became rare due to rapid deforestation caused by over harvesting and exploitative trade of medicinal plants in the forest, like Gloriosa superbaL. (Liliaceae), Gymnema sylvestre R.Br. (Asclepiadaceae), Curculigo orchidioides Gaertn. (Hypoxideae), Aegle marmelos (L.) Corr. (Rutaceae), Buchanania lanzan Spreng. (Anacardiaceae), Dioscorea bulbiferaL. (Dioscoreaceae), Phyllanthus emblica L. (Euphorbiaceae), Plambago zeylanica L.(Plumbaginaceae), etc.

The present investigation has recommended to conclude that among the 229 plant species most dominating species belong to Poaceae family and dominating trees in the Ganpur forest are Shorea robusta Gaertn.f. (Dipterocarpaceae), Madhuca indica J.F.Gmel. (Sapotaceae), Terminalia bellerica Roxb. (Combretaceae), and Acacia auriculiformis (Mimosaceae). Among the collected species 112 species are recognized as medicinally important to the villagers and those species gradually became threatened.

The study also concludes that the persistency of the ethnomedicine practices in the surrounding villages of the forest is still dependent on indigenous knowledge for their health care that are providing a 
cheaper and accessible alternative to the high cost pharmaceutical remedies. Ethnomedicinal knowledge of the tribal people of the surrounding areas of the forest are very rich but it has also been noticed that only the aged people are able to describe the mode of use of the traditional plant medicine. Therefore, it can be concluded that the young generations are not interested to cultivate the indigenous knowledge of preparing medicine from different plants. So, it is a great threat for nature to maintain the balance of biodiversity and as well as for conservation of the different plant species. The tribes who depend on forest wealth are the real custodians that safeguard the medicinal plants till now. Rapid deforestation caused by over harvesting and exploitative trade of medicinal plants has significantly reduced the availability of the medicinal plants in the forest. Local healers and healers from neighbouring state Jharkhand is identified as one of the major threats to vegetation and flora of the forest.

The main purposes of the study was to document and to prepare a database for the use of the plant species in the forest as well as the diversity of the forest. Now it is very much necessary that the Government should take an important role to nurture the traditional knowledge of preparation ethno-medicine from plants for different diseases, and then only it will be possible to save lots of plant species for future benefit of the nation.

\section{Acknowledgements:}

The authors are thankful to the tribal guru Jatha Soren,Sibu Mardi and others, the villagers, the Bit Officer Mr.Pradip Kumar Kundu of Ganpur Forest Range,Birbhum,West Bengal, and the
Department of Botany, Visva-Bharati, who co-operated to carry out the present investigation of works. Thanks are also to the Head, Department of Botany for necessary laboratory facilities.

\section{References}

Baily,L.H. (1949).Manual of cultivated plants.ed.2,New York.

Basak, R. K. and Guha Bakshi, D. N. (1977): Floristic studies on the lower Ganga plain of the state of West Bengalpresent status and future strategies. Bull. Bot. Surv. India 19:42-55.

Basak, R.K. (1979): Some noteworthy plants from Birbhum district,West Bengal.Indian For.105(6):471-481.

Basak,R.K. (1968): A note on the distribution of some plants in Birbhum district,West Bengal,Bull.Bot Surv.India 10:254-257.

Basak,R.K. (1975) :Flora (communicated by the Botanical Survey of India) in D.Majumder Ed. West Bengal District Gazetteer: Birbhum ; 40-47, Calcutta.

Basak,R.K. (1979) : Some noteworthy plants from Birbhum district ,West Bengal,Indian forester; 105(6),477-481

Bor,N.L. (1953). Manual of Indian Forest Botany.Oxford University Press. London.

Bor,N.L. (1960).Grasses of Burma, Ceylon, India and Pakistan (excluding Bambuseae). London.

Champion, H.S. and Seth, S.K. (1968). A revised survey of forest types of India. Dehradun, India: Manager of publication.

Chopra,R.N., Nayar,S.L. and Chopra,I.C. (1956).Glossary of Medicinal Plants. C.S.I.R. New Delhi.

Datta, B.D. and Mandal, S. (1998). A contribution to the forest trees of Birbhum district, W. B. (India). J. Econ. Taxon. Bot. 22(3): 537-545.

Guha,B.P. (1968): An account of the floristic survey of Birbhum district(West Bengal) I (RananculaceaeMoringaceae), Bull.Bot.Soc.Bengal 22:109-121.I fig. 166 spp. listed. 
concise introd. chapter.

Guha,B.P. (1971): Grasses and sedges of Birbhum (West Bengal) Bull. Bot. Soc.Bengal 25:5-18

Hooker,J.D. (1872-97). The Flora of British India. 7 vols. London.

Jain, S.K (1991). Dictionary of Indian Folk Medicine and Ethnobotany. Deep Publication,New Delhi.

Jain, S.K and Rao, R.R, (1977).A Hand Book of Field and Herbarium Methods, Todays \& Tomorrows Publication, New Delhi.

Jain, S.K. (1981). Glimpsesn of Indian EthnoBotany.Oxford and IBH Publishing House.

Lawrence, G.H.M. (1951). Taxonomy of Vascular Plants, Macmillan Company, New York.

Linnaeus, C. (1753). Species Plantarum. Vols.2. Stockholm (Facsimile ed.London, 1957-1959).

Maity, G.G. and D.N.Guha Bakshi. (1981), Invasion of Exotic Weeds in West Bengal since 1903. Dicotyledons and Monocotyledons J.Econ.Tax.Bot.2:121.

Majee, S., Mondal, S. and Mandal, S. (1998). Observations on the plant wealth of Purulia district, West Bengal with reference to aerobiology. Environm. Ecol. 16(3):501-513.

Manilal,K.S. and Sivarajan, V.V. (1982). Flora of Calicut. Bishen Singh Mahendra pal Singh; Dehra Dun, India.

Mukhopadhayay,C.R. (1987): Aquatic and semi-aquatic plants of Birbhum district, West Bengal. J.Econ. Tax. Bot. 9910:230-238.

Nayar ,M.P. (1987).In situ conservation of wild flora resources. Bull.Bot. Surv. India. 29:319-333.

Rahman, C.H and Mandal, S. (1999).New records of some exotic plant species from Birbhum district,West Bengal. Indian Journal of Applied and Pure Biology 14(2) 150-156.

Rahman, C.H, Ghosh, A. and Mandal, S. (2008). Studies on ethnomedicinal uses of plants by the tribals of Birbum district, West Bengal.Indian Journal of Environment and Ecoplanning 15(12)71-78.

Sahu, S.C., Dhal, N.K. and Mohanty R.C. (2012):Tree species diversity, distribution and population structure in a tropical dry deciduous forest of Malygiri hill ranges,Eastern India.Tropical Ecology 53(2),163-168.

Santapau, H. (1955). The Botanical Collectors Mannual: a handbook. New Delhi.

Sanyal, M.N. (1994). Flora of Bankura district, West Bengal,Bishen Sing Mahendra Pal Sing,Dehra Dun, India.

Takhtajan, A. (1969). Flowering Plants-origin and dispersal,Smithsonian Institute Press, Washington.

Varma,S.K. (1981). Flora of Bhagalpur (Dicotyledons).Today and Tomorrow's Printers and Publishers; New Delhi, India.

\section{How to cite this article:}

Nisith Ranjan Sarkar, Subrata Mondal and Sudhendu Mandal. 2016. Phytodiversity of Ganpur forest, Birbhum district, West Bengal, India with reference to their medicinal properties. Int.J.Curr.Microbiol.App.Sci. 5(6): 973-989.

doi: http://dx.doi.org/10.20546/ijcmas.2016.506.104 